\title{
Raising and Lowering Operators for Askey-Wilson Polynomials ${ }^{\star}$
}

\author{
Siddhartha SAHI
}

Department of Mathematics, Rutgers University, New Brunswick, NJ 08903, USA

E-mail:sahi@math.rutgers.edu

Received September 20, 2006, in final form December 27, 2006; Published online January 04, 2007

Original article is available at http://www.emis.de/journals/SIGMA/2007/002/

\begin{abstract}
In this paper we describe two pairs of raising/lowering operators for AskeyWilson polynomials, which result from constructions involving very different techniques. The first technique is quite elementary, and depends only on the "classical" properties of these polynomials, viz. the $q$-difference equation and the three term recurrence. The second technique is less elementary, and involves the one-variable version of the double affine Hecke algebra.
\end{abstract}

Key words: orthogonal polynomials; Askey-Wilson polynomials; q-difference equation; three term recurrence; raising operators; lowering operators; root systems; double affine Hecke algebra

2000 Mathematics Subject Classification: 33D45; 33D52; 33D80

Dedicated to the memory of Vadim Kuznetsov

\section{Introduction}

One of the approaches to proving integrality of the $(q, t)$-Kostka coefficients is the idea, due to Kirillov-Noumi $[9,10]$ and Lapointe-Vinet [16, 17], of using raising operators for Macdonald polynomials. (See also [6, 7, 11, 21] for other approaches.) In their proof Kirillov and Noumi give an explicit construction of such raising operators for the Macdonald polynomials $J_{\lambda}(x ; q, t)$ for the root system of type $A_{n-1}$. They also pose the problem of finding analogous operators for the six-parameter Koornwinder corresponding to the $B C_{n}$ root system.

This question was also raised by Tom Koornwinder at the Edinburgh conference on symmetric functions organized by Vadim Kuznetsov. The case $n=1$ corresponds to the celebrated AskeyWilson polynomials and Koornwinder's paper [14] from that conference contains partial results in this direction as well as a survey of earlier results.

In this paper we such construct raising/lowering operators for Askey-Wilson polynomials. In fact we describe two such pairs of operators, which result from constructions involving very different techniques.

The first technique is quite elementary, and depends only on the "classical" properties of these polynomials, viz. the $q$-difference equation and the three term recurrence. Therefore it can be applied to all the polynomials in the Askey scheme. After this work was completed, we obtained a recent preprint by T. Koornwinder [15], the main result of which is very close to this approach. Also through [15] we discovered still earlier work of G. Bangerezako [2] which obtains similar operators based on an ad-hoc factorization of the Askey-Wilson operator. Our approach however is more direct and quite short.

*This paper is a contribution to the Vadim Kuznetsov Memorial Issue "Integrable Systems and Related Topics". The full collection is available at http://www.emis.de/journals/SIGMA/kuznetsov.html 
The second technique is less elementary and involves the one-variable version of the powerful Hecke algebra method as described in $[18,19,20,22,23,24]$. This approach is related to a fairly remarkable mathematical object - the double affine Hecke algebra (see [3, 4, 5, 22]). The calculations, while non-trivial to carry out, are conceptually rather straightforward. The raising/lowering operators so obtained are different from those coming from the "classical" method. This method also provides a new factorization of the Askey-Wilson operator described in Lemma 3, which is much simpler than that of Bangerezako.

In subsequent work, we hope to extend these methods to construct raising operators for Koornwinder polynomials [13, 25, 22, 23].

\section{The classical approach}

\subsection{Askey Wilson polynomials}

The $q$-hypergeometric series is given by the formula

$$
{ }_{r} \phi_{s}\left(\begin{array}{c}
a_{1}, \ldots, a_{r} \\
b_{1}, \ldots, b_{s}
\end{array} \mid q ; y\right)=\sum_{k \geq 0} \frac{\left(a_{1}, \ldots, a_{r}\right)_{k}}{\left(b_{1}, \ldots, b_{s}\right)_{k}}(-1)^{(1+s-r) k} q^{(1+s-r)\left(\begin{array}{c}
k \\
2
\end{array}\right)} \frac{y^{k}}{(q)_{k}}
$$

where the " $q$-Pochhammer symbols" are defined by

$$
\begin{aligned}
& (a, b, c, \ldots)_{k}:=(a)_{k}(b)_{k}(c)_{k} \cdots, \\
& (a)_{k}:=(1-a)(1-a q) \cdots\left(1-a q^{k-1}\right) .
\end{aligned}
$$

The Askey-Wilson polynomials [1] are defined by the formula

$$
P_{n}(z ; a, b, c, d \mid q)=\frac{(a b, a c, a d)_{n}}{a^{n}\left(a b c d q^{n-1}\right)_{n}}{ }_{4} \phi_{3}\left(\begin{array}{c}
q^{-n}, a b c d q^{n-1}, a z, a z^{-1} \\
a b, a c, a d
\end{array} \mid q ; q\right) .
$$

Since $\left(q^{-n}\right)_{k}$ vanishes for $k>n$, we have

$$
{ }_{4} \phi_{3}(\cdots)=\sum_{k=0}^{n}\left[\frac{\left(a b c d q^{n-1}\right)_{k}}{(a b, a c, a d)_{k}}\right]\left[\frac{\left(q^{-n}\right)_{k} q^{k}}{(q)_{k}}\right](a z)_{k}\left(a z^{-1}\right)_{k} .
$$

It follows that $P_{n}$ is a Laurent polynomial of degree $n$, which is moreover symmetric in $z$ and $z^{-1}$ and is of the form

$$
P_{n}(z ; a, b, c, d \mid q)=\left(z^{n}+z^{-n}\right)+\text { lower terms. }
$$

It is also symmetric in $\{a, b, c, d\}$ although this is not entirely obvious from the formula above.

We have chosen to normalize $P_{n}$ in order to make it monic. Of course there are several other possible normalizations, and we discuss some of these below.

First of all, we remark that formula (3.1.7) of [12] considers the polynomial

$$
\frac{(a b, a c, a d)_{n}}{a^{n}}{ }_{4} \phi_{3}\left(\begin{array}{c}
q^{-n}, a b c d q^{n-1}, a z, a z^{-1} \\
a b, a c, a d
\end{array} \mid q ; q\right)
$$

which is $\left(a b c d q^{n-1}\right)_{n}$ times our $P_{n}$.

Next, since the Askey-Wilson polynomial is symmetric in $z, z^{-1}$, it can be expressed as an (ordinary) polynomial of degree $n$ in

$$
x=\left(z+z^{-1}\right) / 2 .
$$


The function $p_{n}(x)$ considered in (3.1.5) of [12], is monic in $x$, and hence is related to our normalization $P_{n}$ by the formula

$$
p_{n}\left(\frac{z+z^{-1}}{2}\right)=2^{-n} P_{n}(z)
$$

Finally, the polynomials $P_{n}$ are orthogonal with respect the inner product $\langle\cdot, \cdot\rangle$ defined in (3.1.2) of [12]. If we define

$$
Q_{n}=\gamma_{n} P_{n},
$$

where

$$
\gamma_{n}=\frac{\left(a b q^{n}, a c q^{n}, a d q^{n}, b c q^{n}, b d q^{n}, c d q^{n}, q^{n+1}\right)_{\infty}}{\left(a b c d q^{2 n}\right)_{\infty}}\left(a b c d q^{n-1}\right)_{n} .
$$

Then $Q_{n}$ is dual to $P_{n}$ in the sense that

$$
\left\langle P_{m}, Q_{n}\right\rangle=\delta_{m, n}
$$

\subsection{Raising and lowering operators}

The main result of this section are the following raising and lowering operators for the AskeyWilson polynomials:

Theorem 1. For all $n>1$, the Askey-Wilson polynomials satisfy the relations

$$
\begin{aligned}
& {\left[D\left(z+z^{-1}\right)-\lambda_{n-1}\left(z+z^{-1}\right)-\alpha_{n}\left(\lambda_{n}-\lambda_{n-1}\right)\right] P_{n}=\left(\lambda_{n+1}-\lambda_{n-1}\right) P_{n+1},} \\
& {\left[D\left(z+z^{-1}\right)-\lambda_{n+1}\left(z+z^{-1}\right)-\alpha_{n}\left(\lambda_{n}-\lambda_{n+1}\right)\right] Q_{n}=\left(\lambda_{n-1}-\lambda_{n+1}\right) Q_{n-1},}
\end{aligned}
$$

where $D, \lambda_{n}$ and $\alpha_{n}$ are as in (3) and (6) below.

Proof. The proof involves two key properties of the Askey-Wilson polynomials.

The first property is the ' $q$-difference equation' from (3.1.7) ) of [12] which asserts that $P_{n}$ is an eigenfunction for the Askey-Wilson operator, i.e.

$$
D P_{n}(z)=\lambda_{n} P_{n}(z)
$$

The operator and its eigenvalue are defined by

$$
\begin{aligned}
& D=A(z)\left(T_{q}-1\right)+A\left(z^{-1}\right)\left(T_{q^{-1}}-1\right), \\
& \lambda_{n}=\left(q^{-n}-1\right)\left(1-a b c d q^{n-1}\right)=\left(q^{-n}+a b c d q^{n-1}\right)-\left(1+a b c d q^{-1}\right),
\end{aligned}
$$

where $A(z)$ is the rational function

$$
A(z)=\frac{(a z, b z, c z, d z)_{1}}{\left(z^{2}\right)_{2}}=\frac{(1-a z)(1-b z)(1-c z)(1-d z)}{\left(1-z^{2}\right)\left(1-q z^{2}\right)}
$$

and $T_{q}$ is the shift operator

$$
T_{q} f(z)=f(q z) .
$$

(To forestall possible confusion we emphasize that, in accordance with custom, we think of $f(z)$ as a Laurent polynomial rather than as a function of $z$. This means that we have $T_{q}\left(z^{k}\right)=q^{k} z^{k}$ rather than $T_{q}\left(z^{k}\right)=q^{-k} z^{k}$.) 
The second key property of these polynomials is the 'normalized recurrence relation' from (3.1.5) of [12] which can be rewritten in the form

$$
\left(z+z^{-1}\right) P_{n}=P_{n+1}+\alpha_{n} P_{n}+\frac{\gamma_{n-1}}{\gamma_{n}} P_{n-1} \quad \text { for } \quad n>1,
$$

where

$$
\alpha_{n}=a+1 / a-\frac{a\left(b c q^{n-1}, b d q^{n-1}, c d q^{n-1}, q^{n}\right)_{1}}{\left(a b c d q^{2 n-2}\right)_{2}}-\frac{\left(a b q^{n}, a c q^{n}, a d q^{n}, a b c d q^{n-1}\right)_{1}}{a\left(a b c d q^{2 n-1}\right)_{2}} .
$$

We combine these two properties as follows:

First apply the operators $D-\lambda_{n-1}$ and $D-\lambda_{n+1}$, respectively, to the recurrence relation to get

$$
\begin{aligned}
& \left(D-\lambda_{n-1}\right)\left(z+z^{-1}-\alpha_{n}\right) P_{n}=\left(\lambda_{n+1}-\lambda_{n-1}\right) P_{n+1} \\
& \left(D-\lambda_{n+1}\right)\left(z+z^{-1}-\alpha_{n}\right) P_{n}=\frac{\gamma_{n-1}}{\gamma_{n}}\left(\lambda_{n-1}-\lambda_{n+1}\right) P_{n-1} .
\end{aligned}
$$

Finally simplify, using the $q$-difference equation (2), to get

$$
\begin{aligned}
& {\left[D\left(z+z^{-1}\right)-\lambda_{n-1}\left(z+z^{-1}\right)-\alpha_{n}\left(\lambda_{n}-\lambda_{n-1}\right)\right] P_{n}=\left(\lambda_{n+1}-\lambda_{n-1}\right) P_{n+1},} \\
& {\left[D\left(z+z^{-1}\right)-\lambda_{n+1}\left(z+z^{-1}\right)-\alpha_{n}\left(\lambda_{n}-\lambda_{n+1}\right)\right] Q_{n}=\left(\lambda_{n-1}-\lambda_{n+1}\right) Q_{n-1}}
\end{aligned}
$$

as desired.

\section{The Hecke algebra approach}

In this section we provide raising/lowering operators for Askey-Wilson polynomials based on Hecke algebra considerations $[22,23]$. Once again the main idea is quite straightforward, although the calculations are a little more intricate. The resulting formulas are different and perhaps slightly simpler.

\subsection{The Hecke algebra}

The key to this approach are the involutions $s_{1}, s_{0}$ which act on Laurent polynomials as follows:

$$
s_{1} f(z)=f\left(z^{-1}\right) \quad \text { and } \quad s_{0} f(z)=f\left(q z^{-1}\right) .
$$

Once again we regard these operators as acting on polynomials, rather than functions, so that we have

$$
s_{1}\left(z^{k}\right)=z^{-k} \quad \text { and } \quad s_{0}\left(z^{k}\right)=q^{k} z^{-k} .
$$

These operators provide a factorization of the $q$-shift operators, and one has

$$
s_{1} s_{0}=T_{q} \quad \text { and } \quad s_{0} s_{1}=T_{q^{-1}} .
$$

The affine Hecke algebra $[22,23]$ is the algebra of operators generated by the two operators $T_{0}$ and $T_{1}$ defined as

$$
T_{i}:=t_{i}+r_{i}\left(s_{i}-1\right),
$$

where

$$
\begin{aligned}
& t_{0}=-c d / q, \quad r_{0}=\frac{(z-c)(z-d)}{\left(z^{2}-q\right)}, \\
& t_{1}=-a b, \quad r_{1}=\frac{(1-a z)(1-b z)}{\left(1-z^{2}\right)} .
\end{aligned}
$$


Remark 1. The operator $T_{i}$ as defined here is $t_{i}^{1 / 2}$ times the corresponding operator from [22, 23]. This accounts for the slight difference between the formulas here and in [23].

From the definition of $T_{1}$ it follows that a polynomial $f$ is symmetric in $z, z^{-1}$, if and only if

$$
T_{1} f=t_{1} f .
$$

Consequently, if $g$ is any polynomial, then the quadratic relation (9) implies that $\left(T_{1}+1\right) g$ is a symmetric polynomial. The operators $T_{i}$ are deformations of $s_{i}$ and satisfy a quadratic relation. For the convenience of the reader unfamiliar with the Hecke algebra, we give a proof this relation.

Lemma 1. The operators $T_{i}$ satisfy the relation

$$
\left(T_{i}-t_{i}\right)\left(T_{i}+1\right)=0 .
$$

Proof. Define $s_{i}\left(r_{i}\right)=r_{i}^{\prime}$, then we claim that

$$
r_{i}+r_{i}^{\prime}=t_{i}+1
$$

To see this, we calculate for $i=0$,

$$
\begin{aligned}
r_{0}+r_{0}^{\prime} & =\frac{(z-c)(z-d)}{\left(z^{2}-q\right)}+\frac{\left(q z^{-1}-c\right)\left(q z^{-1}-d\right)}{\left(q^{2} z^{-2}-q\right)} \\
& =\frac{(z-c)(z-d)}{\left(z^{2}-q\right)}-\frac{(q-c z)(q-d z)}{q\left(z^{2}-q\right)} \\
& =\frac{q\left(z^{2}-c z-d z+c d\right)-\left(q^{2}-q c z-q d z+c d z^{2}\right)}{q\left(z^{2}-q\right)} \\
& =\frac{q z^{2}+q c d-q^{2}-c d z^{2}}{q\left(z^{2}-q\right)}=\frac{(q-c d)\left(z^{2}-q\right)}{q\left(z^{2}-q\right)} \\
& =1-\frac{c d}{q}=1+t_{0} .
\end{aligned}
$$

The calculation for $i=1$ is similar and simpler.

Now the quadratic relation can be proved as follows:

$$
\begin{aligned}
\left(T_{1}-t_{1}\right)\left(T_{1}+1\right) & =r_{i}\left(s_{i}-1\right)\left[t_{i}+1+r_{i}\left(s_{i}-1\right)\right] \\
& =r_{i}\left[\left(t_{i}+1\right)\left(s_{i}-1\right)+\left(s_{i} r_{i}\right)\left(s_{i}-1\right)-r_{i}\left(s_{i}-1\right)\right] \\
& =r_{i}\left[\left(t_{i}+1\right)\left(s_{i}-1\right)+\left(r_{i}^{\prime} s_{i}\right)\left(s_{i}-1\right)-r_{i}\left(s_{i}-1\right)\right] \\
& =r_{i}\left[\left(t_{i}+1\right)\left(s_{i}-1\right)+r_{i}^{\prime}\left(1-s_{i}\right)-r_{i}\left(s_{i}-1\right)\right] \\
& =r_{i}\left(t_{i}+1-r_{i}-r_{i}^{\prime}\right)\left(s_{i}-1\right) \stackrel{\text { by } \stackrel{(10)}{=} 0 .}{ } .
\end{aligned}
$$

The following result is an immediate consequence

Corollary 1. The operators $T_{i}$ are invertible, with

$$
t_{i} T_{i}^{-1}=T_{i}-t_{i}+1 .
$$

We will also need a number of commutation results between the $T_{i}$ and the multiplication operator by $z$. They follow directly from the definition, and we leave the (easy) proof to the reader.

Lemma 2. The operators $T_{i}$ satisfy the following commutation relations

$$
\begin{aligned}
& z t_{0} T_{0}^{-1}=q T_{0} z^{-1}+c+d, \\
& \left(T_{1}+1\right) z^{-1}=t_{1} z^{-1}+z T_{1}+a+b, \\
& \left(T_{1}+1\right) z=z+t_{1} z^{-1} T_{1}^{-1}-(a+b) .
\end{aligned}
$$




\subsection{Nonsymmetric Askey-Wilson polynomials}

The next ingredient in the Hecke algebra method are the nonsymmetric Askey-Wilson polynomials. These are certain Laurent polynomials, $E_{n}, n \in \mathbb{Z}$, which can be characterized up to multiples as eigenfunctions of the operator

$$
Y=T_{1} T_{0}
$$

More precisely, one has

$$
Y E_{n}=\mu_{n} E_{n}
$$

where

$$
\mu_{n}= \begin{cases}q^{n} & \text { for } n<0 \\ q^{n} t_{1} t_{0}=q^{n-1} a b c d & \text { for } \quad n \geq 0\end{cases}
$$

The symmetric Askey-Wilson polynomials $P_{|n|}$ are closely related to $E_{ \pm n}$. Up to normalization, one has $P_{0}=E_{0}=1$, while for $n>0$ we have up to a scalar

$$
P_{|n|} \sim\left(T_{1}+1\right) E_{ \pm n}=c_{n}^{ \pm} E_{n}+c_{-n}^{ \pm} E_{-n} .
$$

The explicit formula for the coefficients $c_{n}^{ \pm}$and $c_{-n}^{ \pm}$is known, but will not be needed in what follows.

We now define a slight variant of the Askey-Wilson operator, as follows:

$$
D^{\prime}=A(z)\left(T_{q}-s_{1}\right)+A\left(z^{-1}\right)\left(T_{q^{-1}} s_{1}-1\right) .
$$

Observe that $D$ and $D^{\prime}$ have the same action on functions which are symmetric $z$ and $z^{-1}$, thus the Askey-Wilson polynomials satisfy

$$
D^{\prime} P_{n}=D P_{n}=\lambda_{n} P_{n} .
$$

Just as the operator $s_{0}$ and $s_{1}$ factorize the $q$-shift operator, it turns out that the operators $T_{0}$ and $T_{1}$ provide a factorization of $D^{\prime}$.

Lemma 3. The operator $D^{\prime}$ of formula (14) admits the following factorization:

$$
D^{\prime}=\left(T_{1}+1\right)\left(T_{0}-t_{0}\right) .
$$

Proof. To prove this, we calculate as follows

$$
\begin{aligned}
\left(T_{1}+1\right)\left(T_{0}-t_{0}\right) & =\left(t_{1}+r_{1}\left(s_{1}-1\right)+1\right)\left(r_{0}\left(s_{0}-1\right)\right) \stackrel{\text { by }(10)}{=}\left(r_{1}^{\prime}+r_{1} s_{1}\right)\left(r_{0} s_{0}-r_{0}\right) \\
& =r_{1}^{\prime} r_{0}\left(s_{0}-1\right)+r_{1} \widetilde{r}_{0}\left(s_{1} s_{0}-s_{1}\right)=r_{1}^{\prime} r_{0}\left(T_{q}^{-1} s_{1}-1\right)+r_{1} \widetilde{r}_{0}\left(T_{q}-s_{1}\right),
\end{aligned}
$$

where $r_{1}^{\prime}=r_{1}\left(z^{-1}\right)$ and $\widetilde{r}_{0}=r_{0}\left(z^{-1}\right)$.

Comparing the formulas for $r_{i}(7)$ and $A(z)$ (4), we conclude that

$$
A(z)=r_{1} \widetilde{r}_{0} \quad \text { and } \quad A\left(z^{-1}\right)=r_{1}^{\prime} r_{0}
$$

which completes the proof. 


\subsection{Raising and lowering operators}

To state our main result we need some notation. We write

$$
e_{1}=a+b+c+d, \quad e_{3}=a b c+a b d+a c d+b c d .
$$

Also recall that for $n \geq 0, \lambda_{n}$ is the symmetric Askey-Wilson eigenvalue as in (3). For $n<0$ we define

$$
\lambda_{n}=\lambda_{|n|}
$$

and for all integral $n$ we set

$$
\beta_{n}=\frac{\lambda_{n}+1-\mu_{n-1}}{\mu_{n-1}-\mu_{-n}} e_{1}-\frac{1-\mu_{n-1}}{\mu_{n-1}-\mu_{-n}} e_{3}
$$

Theorem 2. The Askey-Wilson polynomials satisfy the following relations:

$$
\begin{aligned}
& {\left[D^{\prime} z+\left(1-q^{1-n}\right)\left(z+z^{-1}\right)+\beta_{-n}\right] P_{n}=\left(q^{n} a b c d-q^{1-n}\right) P_{n+1}, \quad n \geq 0,} \\
& {\left[D^{\prime} z+\left(1-q^{n} a b c d\right)\left(z+z^{-1}\right)+\beta_{n}\right] Q_{n}=\left(q^{1-n}-q^{n} a b c d\right) Q_{n-1}, \quad n>0 .}
\end{aligned}
$$

The key for the proof is the "affine intertwiner" for the nonsymmetric Askey-Wilson polynomials from [23]. This involves the additional parameters $u_{0}$ and $u_{1}$, which satisfy the relations

$$
a=t_{1}^{1 / 2} u_{1}^{1 / 2}, \quad b=-t_{1}^{1 / 2} u_{1}^{-1 / 2}, \quad c=q^{1 / 2} t_{0}^{1 / 2} u_{0}^{1 / 2}, \quad d=-q^{1 / 2} t_{0}^{-1 / 2} u_{0}^{1 / 2} .
$$

Now from Theorem 1.2 of [23] we have, up to a multiple,

$$
E_{n} \sim\left(a_{n} U_{0}+b_{n}\right) E_{-n-1},
$$

where

$$
a_{n}=\left(q^{\bar{n}}-q^{\overline{-n-1}}\right) \quad \text { and } \quad b_{n}=q^{\bar{n}}\left(u_{0}^{-1 / 2}-u_{0}^{1 / 2}\right)+q^{-1 / 2}\left(u_{1}^{-1 / 2}-u_{1}^{1 / 2}\right)
$$

with

$$
q^{\bar{n}}=\mu_{n} t_{1}^{1 / 2} t_{0}^{1 / 2}
$$

and $U_{0}$ is the operator

$$
U_{0}=q^{-1 / 2} t_{0}^{1 / 2} T_{0}^{-1} z .
$$

We will derive Theorem 2 from formula (19); however some remarks are in order before we proceed:

1. There is a typo in the statement of formula (19) in Theorem 1.2 of [23], namely $n$ and $-n-1$ have been inadvertently switched. This is easily seen by comparison with Theorem 4.1 from which Theorem 1.2 is derived.

2. The formula for $U_{0}$ here is slightly different from that in [23] because of the difference in $T_{0}$ - see Remark 1).

3. Although Theorem 1.2 in [23] is only stated (and needed) for $n \geq 0$, it is easy to see that after the correction above it holds for all integer $n$. 
4. Finally, we note that the ideas of [22] and [23] work in the more general setting of Koornwinder polynomials, and they involve the affine intertwiner $S_{0}$, which can also be written as

$$
S_{0}=\left[Y, z^{-1} T_{1}^{-1}\right]
$$

It is expected that this operator will play a key role in the raising operators for Koornwonder polynomials.

We now give the proof of Theorem 2 .

Proof. We first simplify (19) by multiplying through by $q^{1 / 2} t_{1}^{1 / 2} t_{0}$. This gives

$$
\begin{aligned}
E_{n} & \sim\left[\left(\mu_{n}-\mu_{-n-1}\right) t_{0} T_{0}^{-1} z-\mu_{n}(c+d)-t_{0}(a+b)\right] E_{-n-1} \\
& \sim\left[t_{0} T_{0}^{-1} z-\frac{\mu_{n}(c+d)+t_{0}(a+b)}{\mu_{n}-\mu_{-n-1}}\right] E_{-n-1} .
\end{aligned}
$$

Replacing $n$ by $n-1$, we get

$$
E_{n-1} \sim\left[t_{0} T_{0}^{-1} z-\frac{\mu_{n-1}(c+d)+t_{0}(a+b)}{\mu_{n-1}-\mu_{-n}}\right] E_{-n} .
$$

For ease in subsequent calculations, we write this as

$$
E_{n-1} \sim\left(t_{0} T_{0}^{-1} z-\kappa_{n}\right) E_{-n},
$$

where

$$
\kappa_{n}=\frac{\mu_{n-1} y+t_{0} x}{\mu_{n-1}-\mu_{-n}}, \quad x=a+b, \quad y=c+d .
$$

The key idea to obtain a raising operator for $P_{n}$ is as follows: By formula (13), for $n>1, P_{|n|}$ is a combination of $E_{n}$ and $E_{-n}$. We first kill off the $E_{n}$ component. This can be accomplished by applying the operator $Y-\mu_{n}$ to $P_{n}$. However it is more convenient (and equivalent) to apply the operator

$$
t_{1} t_{0}\left(Y^{-1}-\mu_{n}^{-1}\right)=t_{1} t_{0} T_{0}^{-1} T_{1}^{-1}-\frac{t_{1} t_{0}}{\mu_{n}}
$$

For $n \neq 0$ we have $\frac{t_{1} t_{0}}{\mu_{n}}=\mu_{-n}$. Thus since $P_{n}$ is symmetric, formula (8) implies that up to a non-zero multiple, one has

$$
\left(t_{0} T_{0}^{-1}-\mu_{-n}\right) P_{n} \sim E_{-n} .
$$

Although the argument given above only applies for $n \neq 0$, it is easy to see that formula (22) is true (up to a non-zero multiple) for $n=0$ as well! Now combining formulas (13), (20), and (22) we conclude that up to a multiple, we have

$$
P_{|n-1|} \sim R_{n} P_{|n|},
$$

where

$$
R_{n}=\left(T_{1}+1\right)\left(t_{0} T_{0}^{-1} z-\kappa_{n}\right)\left(t_{0} T_{0}^{-1}-\mu_{-n}\right) .
$$

The main problem now is to simplify the expression of the operator $R_{n}$ using properties of $P_{|n|}$. 
We first calculate using Lemma 2 and (11), as follows

$$
\begin{aligned}
\left(t_{0} T_{0}^{-1} z-\kappa_{n}\right)\left(t_{0} T_{0}^{-1}-\mu_{-n}\right) \\
\quad=t_{0} T_{0}^{-1} z t_{0} T_{0}^{-1}-t_{0} T_{0}^{-1}\left(\mu_{-n} z+\kappa_{n}\right)+\kappa_{n} \mu_{-n} \\
\quad=t_{0} T_{0}^{-1}\left(q T_{0} z^{-1}+y\right)-t_{0} T_{0}^{-1}\left(\mu_{-n} z+\kappa_{n}\right)+\kappa_{n} \mu_{-n} \\
\quad=t_{0} T_{0}^{-1}\left(y-\mu_{-n} z-\kappa_{n}\right)+q t_{0} z^{-1}+\kappa_{n} \mu_{-n} \\
\quad=\left(T_{0}-t_{0}+1\right)\left(y-\mu_{-n} z-\kappa_{n}\right)+\left(q t_{0} z^{-1}+\kappa_{n} \mu_{-n}\right) .
\end{aligned}
$$

Applying $T_{1}+1$ to this, we get by Lemma 3

$$
R_{n}=\left(D^{\prime}+T_{1}+1\right)\left(-\mu_{-n} z+y-\kappa_{n}\right)+\left(T_{1}+1\right)\left(q t_{0} z^{-1}+\kappa_{n} \mu_{-n}\right) .
$$

To simplify this further we note that the commutation relations of Lemma 2 can be rewritten as and hence imply

$$
\begin{aligned}
& \left(T_{1}+1\right) z^{-1}=t_{1} z^{-1}+z T_{1}+x, \\
& \left(T_{1}+1\right) z=z+t_{1} z^{-1} T_{1}^{-1}-x .
\end{aligned}
$$

Also on $P_{|n|}, D^{\prime}$ acts by $\lambda_{n}$ while $T_{1}$ acts by $t_{1}$. Therefore $R_{n}$ acts by the operator

$$
\begin{gathered}
-\mu_{-n}\left[D^{\prime} z+z+z^{-1}-x\right]+\left(\lambda_{n}+t_{1}+1\right)\left(y-\kappa_{n}\right) \\
+q t_{0}\left[t_{1}\left(z+z^{-1}\right)+x\right]+\left(t_{1}+1\right) \kappa_{n} \mu_{-n} .
\end{gathered}
$$

Dividing by $-\mu_{-n}$, we see that up to a multiple

$$
\left[D^{\prime} z+\left(1-\frac{q t_{1} t_{0}}{\mu_{-n}}\right)\left(z+z^{-1}\right)+\beta_{n}^{\prime}\right] P_{|n|} \sim P_{|n-1|},
$$

where

$$
\beta_{n}^{\prime}=-x-\frac{q t_{0}}{\mu_{-n}} x-\frac{\lambda_{n}+t_{1}+1}{\mu_{-n}}\left(y-\kappa_{n}\right)-\left(t_{1}+1\right) \kappa_{n} .
$$

We now show that $\beta_{n}^{\prime}$ is equal to $\beta_{n}$ from formula (16). For this we simplify the expression, substituting for $\kappa_{n}$, using (20) above to get

$$
\begin{aligned}
\beta_{n}^{\prime} & =-x-\frac{q t_{0}}{\mu_{-n}} x+\frac{\left(\lambda_{n}+t_{1}+1\right)}{\mu_{-n}} \frac{\mu_{-n} y+t_{0} x}{\mu_{n-1}-\mu_{-n}}-\left(t_{1}+1\right) \frac{\mu_{n-1} y+t_{0} x}{\mu_{n-1}-\mu_{-n}} \\
& =\frac{\lambda_{n}+1-\mu_{n-1}}{\mu_{n-1}-\mu_{-n}} y+\frac{1-\mu_{n-1}}{\mu_{n-1}-\mu_{-n}} t_{1} y+c_{1} x+c_{2}\left(q t_{0} x\right),
\end{aligned}
$$

where $c_{1}$ and $c_{2}$ are moderately complicated expressions which can be computed explicitly. However, we can save some computation by observing that since the result is a priori symmetric in $\{a, b, c, d\}, c_{1}$ and $c_{2}$ must reduce to the coefficients of $y$ and $-t_{1} y$ respectively.

It follows then that we have

$$
\begin{aligned}
\beta_{n}^{\prime} & =\frac{\lambda_{n}+1-\mu_{n-1}}{\mu_{n-1}-\mu_{-n}}(x+y)+\frac{1-\mu_{n-1}}{\mu_{n-1}-\mu_{-n}}\left(q t_{0} x+t_{1} y\right) \\
& =\frac{\lambda_{n}+1-\mu_{n-1}}{\mu_{n-1}-\mu_{-n}} e_{1}-\frac{1-\mu_{n-1}}{\mu_{n-1}-\mu_{-n}} e_{3}=\beta_{n} .
\end{aligned}
$$

Replacing $n$ by $-n$, formula (23) becomes

$$
\left[D^{\prime} z+\left(1-\frac{q t_{1} t_{0}}{\mu_{n}}\right)\left(z+z^{-1}\right)+\beta_{-n}\right] P_{|n|} \sim P_{|-n-1|} .
$$


For $n \geq 0$, we have $\mu_{n}=q^{n} t_{1} t_{0}$ and this becomes

$$
\left[D^{\prime} z+\left(1-q^{1-n}\right)\left(z+z^{-1}\right)+\beta_{-n}\right] P_{n} \sim P_{n+1}
$$

which is (17) up to a multiple.

For $n \geq 1$, we have $\mu_{-n}=q^{-n}$ and formula (23) becomes

$$
\left[D^{\prime} z+\left(1-q^{n} a b c d\right)\left(z+z^{-1}\right)+\beta_{n}\right] P_{n} \sim P_{n-1}
$$

which is (18) up to a multiple.

It remains then only to calculate the multiples in (24), (25).

To determine the multiple (24), it suffices to calculate the coefficient of $z^{n+1}$ on the left. For this we divide the left side of (24) by $z^{n+1}$ and take the limit as $z \rightarrow \infty$. This gives

$$
\begin{gathered}
\lim _{z \rightarrow \infty} \frac{1}{z^{n+1}}\left(A(z) q^{n+1} z^{n+1}-A\left(z^{-1}\right) z^{n+1}+\left(1-q^{1-n}\right) z^{n+1}\right) \\
=\frac{a b c d}{q} q^{n+1}-1+1-q^{1-n}=q^{n} a b c d-q^{1-n}
\end{gathered}
$$

which proves formula (17).

To determine the multiple in (25), we rewrite (25) in the form

$$
\left[D^{\prime} z+\left(1-q^{n} a b c d\right)\left(z+z^{-1}\right)+\beta_{n}\right] P_{n}=c_{n}\left(q^{1-n}-q^{n} a b c d\right) \frac{\gamma_{n-1}}{\gamma_{n}} P_{n-1}, \quad n>0
$$

for some unknown constant $c_{n}$. It then suffices to show that $c_{n}=1$. Subtracting this from (17) we have

$$
\begin{aligned}
& {\left[\left(q^{n} a b c d-q^{1-n}\right)\left(z+z^{-1}\right)+\beta_{-n}-\beta_{n}\right] P_{n}} \\
& \quad=\left(q^{n} a b c d-q^{1-n}\right)\left(P_{n+1}+c_{n} \frac{\gamma_{n-1}}{\gamma_{n}} P_{n-1}\right)
\end{aligned}
$$

or

$$
\left(z+z^{-1}\right) P_{n}=P_{n+1}+\frac{\beta_{n}-\beta_{-n}}{q^{n} a b c d-q^{1-n}} P_{n}+c_{n} \frac{\gamma_{n-1}}{\gamma_{n}} P_{n-1} .
$$

Comparing this with the recurrence relation (5) we deduce $c_{n}=1$, which proves formula (18) and completes the proof of the theorem.

We note in passing that comparison with (5) also proves the following identity (which can be verified independently):

$$
\alpha_{n}=\frac{\beta_{n}-\beta_{-n}}{q^{n} a b c d-q^{1-n}} .
$$

\section{Acknowledgements}

We would like to thank the (anonymous) referee for several insightful suggestions which have improved the paper considerably. The referee has also pointed out that one can give an alternative proof of formulas (17) and (18) by combining Theorem 1 with the following identity relating the operators $D$ and $D^{\prime}$ :

$$
\begin{aligned}
& {\left[\left(1-q^{2}\right) D^{\prime} z+q^{2} D\left(z+z^{-1}\right)-q\left(z+z^{-1}\right) D\right] f} \\
& \quad=(1-q)\left[\left(e_{1}-e_{3}\right)-(1-a b c d)\left(z+z^{-1}\right)\right] f
\end{aligned}
$$

which holds for all symmetric Laurent polynomials $f$. 


\section{References}

[1] Askey R., Wilson J., Some basic hypergeometric polynomials that generalize Jacobi polynomials, Mem. Amer. Math. Soc. 319 (1985), 1-53.

[2] Bangerezako G., The factorization method for the Askey-Wilson polynomials, J. Comput. Appl. Math. 107 (1999), 219-232.

[3] Cherednik I., Double affine Hecke algebras, Knizhnik-Zamolodchikov equations, and Macdonald's operators, Int. Math. Res. Not. (1992), no. 9, 171-180.

[4] Cherednik I., Double affine Hecke algebras and Macdonald's conjectures, Ann. of Math. 141 (1995), 191216.

[5] Etingof P., Oblomkov A., Rains E., Generalized double affine Hecke algebras of rank 1 and quantized del Pezzo surfaces, math.QA/0406480.

[6] Garsia A., Remmel R., Plethystic formulas and positivity for $q$, $t$-Kostka coefficients, in Mathematical Essays in Honor of Gian-Carlo Rota, Editors B. Sagan and R. Stanley, Progr. Math. 161 (1998), 245-262.

[7] Garsia A., Tesler G., Plethystic formulas for the Macdonald q,t-Kostka coefficients, Adv. Math. 123 (1996), $144-222$.

[8] Ion B., Sahi S., Triple groups and Cherednik algebras, Contemp. Math. 417 (2006), 183-206, math.QA/0304186.

[9] Kirillov A., Noumi M., q-difference raising operators for Macdonald polynomials and the integrality of transition coefficients, in Algebraic Methods and $q$-Special Functions, CRM Proceedings and Lecture Notes 22 (1999), 227-243, q-alg/9605005.

[10] Kirillov A., Noumi M., Affine Hecke algebras and raising operators for Macdonald polynomials, Duke Math. J. 93 (1998), 1-39, q-alg/9605004.

[11] Knop F., Integrality of two variable Kostka functions, J. Reine Angew. Math. 482 (1997), 177-189, q-alg/9603027.

[12] Koekoek R., Swarttouw R., The Askey-scheme of hypergeometric orthogonal polynomials and its $q$-analogue, Delft University of Technology, Department of Technical Mathematics and Informatics, Report no. 98-17 (1998), http://aw.twi.tudelft.nl/ koekoek/askey/ch3/par1/par1.html.

[13] Koornwinder T., Askey-Wilson polynomials for root systems of type BC, Contemp. Math. 138 (1992), 189-204.

[14] Koornwinder T., Lowering and raising operators for some special orthogonal polynomials, Contemp. Math. 417 (2006), 227-238.

[15] Koornwinder T., The structure relation for Askey-Wilson polynomials, J. Comput. Appl. Math., to appear, math.CA/0601303.

[16] Lapointe L., Vinet L., Creation operators for the Macdonald and Jack polynomials, Lett. Math. Phys. 40 (1997), 269-286.

[17] Lapointe L., Vinet L., Rodrigues formulas for the Macdonald polynomials, Adv. Math. 130 (1997), 261-279, q-alg/9607025.

[18] Macdonald I., Affine Hecke algebras and orthogonal polynomials, Cambridge University Press, 2003.

[19] Noumi M., Macdonald-Koornwinder polynomials and affine Hecke algebras, RIMS Kokyuroku 919 (1995), 44-55 (in Japanese).

[20] Noumi M., Stokman J., Askey-Wilson polynomials: an affine Hecke algebra approach, in Laredo Lectures on Orthogonal Polynomials and Special Functions, Editors R. Alvarez-Nodarse, F. Marcellan and W. Van Assche, Nova Science Publishers, 2004, 111-144, math.QA/0001033.

[21] Sahi S., Interpolation, integrality, and a generalization of Macdonald's polynomials, Int. Math. Res. Not. (1996), no. 10, 457-471.

[22] Sahi S., Nonsymmetric Koornwinder polynomials and duality, Ann. of Math. 150 (1999), 267-282, q-alg/9710032.

[23] Sahi S., Some properties of Koornwinder polynomials, Contemp. Math. 254 (2000), 395-411.

[24] Stokman J., Koornwinder polynomials and affine Hecke algebras, Int. Math. Res. Not. (2000), no. 19, 1005-1042, math.QA/0002090.

[25] van Diejen J., Self-dual Koornwinder-Macdonald polynomials, Invent. Math. 126 (1996), 319-339, q-alg/9507033. 